\title{
Scientific research topic and researcher background and origin: are topics chosen on bases other than science?
}

\section{Letter to Editor}

Scientific research has become more and more an urgent necessity worldwide, and many countries in the world invest great amounts of money in order to discover efficacious therapies for diseases that are still without any radical treatment such as, Alzheimer's disease and cancer. There have been an increasing number of hot research topics in the last few decades, particularly through focusing on traditional medicine, healthy diets, and pathology mechanistic pathways. Obviously, the collaboration between research groups worldwide is a key element for a successful scientific research. Different laboratories in the world have different and complementary knowledge and research tools, which could lead to fruitful investigations through global expertise exchange. However, I have noticed through browsing numerous published scientific papers that there is a certain hindrance in front of this cooperation and science promotion; i.e. that most of research topics have a tendency to be about a natural drug, a food diet, a health problem, etc. that is related to the region or the country where the research is conducted or to the researcher's origin or cultural background. While this could be in favor of research success, it might be on the other side a limitation to science development. In this paper, I draw attention to this important point with illustrative examples, and I clarify the importance of taking into consideration to strengthen scientific research globalization.

Research topics are reflected as titles of published scientific papers and topics of seminars and academic meetings. It is obvious that a research group may tend to work and publish data about a disease, a potential remedy, etc. that is available or of great interest in the research center localization. This could be explained by many reasons such as research economic budget, and the accessibility of research materials such as local natural drugs, in addition to stronger background of researchers about local health problems and environment, etc. For instance a great majority of published papers about Mediterranean diet are published by researchers or research groups from Mediterranean countries. The same is for topics about
Volume 3 Issue 4 - 2015

\author{
Besma Boubertakh',2 \\ 'Laboratory of Animal Models and Human Disease Mechanisms \\ of Chinese Academy of Sciences \& Yunnan Province, Kunming \\ Institute of Zoology, China \\ ${ }^{2}$ University of Chinese Academy of Sciences, China
}

Correspondence: Key Laboratory of Animal Models and Human Disease Mechanisms, Kunming Institute of Zoology and University of Chinese Academy of Sciences, No. 32 Jiaochang Donglu, Kunming 650223, China,

Emailwww.boubertakhbesma@outlook.com

Received: November 09, 2015 | Published: November 18, 2015

Chinese medicine and Ayurveda, which are in major published by researchers from relevant regions or cultures.

It is of vital importance to strengthen scientific cooperation between research groups from different regions in the world. Different research laboratories have different materials and techniques, and different researchers have different expertise and backgrounds according to their regions. Moreover, each region is rich in certain natural drugs and specific characteristics of diseases and environment, hence the necessity of efficient collaboration in order to take benefit from researchers expertise worldwide to be applied on research topics from all regions of the globe.

\section{Acknowledgements}

None.

\section{Conflict of interest}

The author declares no conflict of interest. 